\title{
Rapidly Reversible Organic Crystalline Switch for Conversion of Heat into Mechanical Energy
}

Madushani Dharmarwardana, ${ }^{\mathrm{a},+}$ Srimanta Pakhira, ${ }^{\mathrm{d}+}$ Raymond P. Welch, ${ }^{\mathrm{a},+}$ Carlos Caicedo-Narvaez ${ }^{\mathrm{b}}$, Michael A. Luzuriaga ${ }^{a}$, Bhargav S. Arimillia, Gregory T. McCandless ${ }^{a}$, Babak Fahimi ${ }^{b}$, Jose L. MendozaCortes, ${ }^{\text {ef, }, *}$ Jeremiah J. Gassensmith ${ }^{\mathrm{a}, \mathrm{c}, *}$

${ }^{\mathrm{a}}$ Department of Chemistry and Biochemistry, ${ }^{\mathrm{b}}$ Renewable Energy and Vehicular Technology Laboratory, and ${ }^{\mathrm{c}}$ Department of Biomedical Engineering, The University of Texas at Dallas, 800 West Campbell Road, Richardson, Texas 75080, United States. ${ }^{\mathrm{d}}$ Discipline of Physics, Discipline of Metallurgy Engineering and Materials Science (MEMS) \& Centre for Advanced Electronics (CAE), Indian Institute of Technology Indore (IIT Indore), Simrol, Khandwa Road, Indore-453552, Madhya Pradesh (M.P.), India. ${ }^{e}$ Department of Chemical \& Biomedical Engineering, FAMU-FSU joint College of Engineering, Tallahassee, Florida 32310, United States. ${ }^{\mathrm{f} C u r r e n t}$ address: Department of Chemical Engineering \& Materials Science, Michigan State University, East Lansing, Michigan 48824, United States.

\begin{abstract}
Solid-state thermoelastic behavior - a sudden exertion of an expansive or contractive physical force following a temperature change and phase transition in a solid-state compound - is rare in organic crystals, few are reversible systems, and most of these are limited to a dozen or so cycles before the crystal degrades or they reverse slowly over the course of many minutes or even hours. Comparable to thermosalience, wherein crystal phase changes induce energetic jumping, thermomorphism produces physical work via consistent and near-instantaneous predictable directional force. In this work, we show a fully reversible thermomorphic actuator that is stable at room temperature for multiple years and is capable of actuation for more than two hundred cycles at near ambient temperature. Specifically, the crystals shrink to $90 \%$ of their original length instantaneously upon heating beyond $45^{\circ} \mathrm{C}$ and expand back to their original length upon cooling below $35{ }^{\circ} \mathrm{C}$. Furthermore, the phase transition occurs instantaneously, with little obvious hysteresis, allowing us to create real-time actuating thermal fuses that cycle between on and off rapidly.
\end{abstract}

\section{Introduction}

Materials that can transduce heat into well-defined and directed motion are rare but have a wide range of applications for a diverse array of technologies from microscopic pumps to energy harvesting. For instance, transducing well-defined thermally induced reciprocating actuation simply by taking advantage of the natural temperature fluctuation between day and night cycles is a possible way to harness ambient temperature changes into useful pollution-free electricity. In addition, the ever-increasing interest in the miniaturization of mechanical devices demands new actuating materials that can produce motion in the micron to millimeter regime. While actuators that reversibly operate from electrochemical ${ }^{1-2}$ and photochemical $^{3}$ stimuli are known, the application of thermal expansion (thermoelastic) actuators has been limited by poor mechanical properties, material fatigue, or low coefficients of thermal expansion (CTEs). For instance, metallic aluminum and lead, which have the highest known CTEs in pure metals, are still in the regime of $20 \times 10^{-6} \mathrm{~K}^{-1}$. Thermo-responsive actuating shape-memory polymers have been developed, ${ }^{4-7}$ but they typically require a significant change in the global environmental temperature to actuate. ${ }^{8}$ 
Dynamic crystals are mechanically active single-crystalline entities capable of amplifying small changes in molecular packing into large-scale anisotropic macroscopic motion. ${ }^{9-10}$ These materials have CTEs over an order of magnitude higher than the best pure-metals and undergo phase transitions at rates exceeding $10^{5}$ times faster than non-thermosalient transitions. ${ }^{9,} 11$ This expansion is caused by a diffusionless phase transition that is initiated at crystalline extremities and rapidly propagates throughout the crystal. ${ }^{12-13}$ In some of these systems, expansion can cause a sudden single-crystal-to-single-crystal (SCSC) phase transition with substantial changes in unit cell size, thus providing even greater material expansion. ${ }^{14-23}$ In many of these SCSC thermosalient events, the transitions are typically irreversible and results in their destruction by splitting, ${ }^{24-25}$ jumping, ${ }^{26-27}$ or even dramatically exploding. ${ }^{28}$ These irreversible dynamic crystals have found applications as fuses, ${ }^{29}$ making use of their one-time ability to actuate to break a circuit; however, this irreversibility greatly limits their applications. In contrast to irreversible systems, there are very few thermally actuated reversible systems, ${ }^{30-31}$ and most of these are limited to a dozen or so cycles before the crystal degrades or they reverse slowly over the course of many minutes or even hours. Designing a thermoelastic system that has the rapid expansive force of a thermosalient solid yet can predictably actuate the same way, at hysteresis free rates is complicated by the fact that structure-function relationships in molecular crystals can be difficult to derive and thus a priori synthesis of actuating materials is challenging.

In this report, we introduce a solid state rapidly actuating organic crystal that undergoes a significant and sudden change in size following a SCSC transition with considerable and measurable force. We find it is a fully reversible actuator capable of more than two hundred cycles and room temperature that remains stable on the benchtop for at least three years. Like thermosalient crystals, the phase transition occurs instantaneously, with little evident hysteresis, allowing us to create real-time thermomechanical actuators that cycle an electrical circuit on and off rapidly. We have previously discovered a family of thermosalient molecular crystals based on a naphthalene diimide (NDI) core with alkoxyphenyl substituents (Figure 1a). ${ }^{12}$ We have reported that the single-action thermosalient behavior is tunable across temperature ranges by altering the alkyl chain from four to nine carbons. ${ }^{32}$ Here, we report that, when the alkyl chain is ten linear carbons, the crystals become thermomorphic - the crystals predictably, reversibly, and instantaneously actuate much like a thermosalient system but in a single direction via negative thermal expansion along one axis. Specifically, the crystals shrink to $90 \%$ of their original length upon heating beyond $45{ }^{\circ} \mathrm{C}$ and expand back to their original length upon cooling below $35^{\circ} \mathrm{C}$. This temperature regime is important because it occurs at just the minimum temperature that can cause a burn in a finite amount of time, giving rise to possible bioengineering applications. ${ }^{33}$

\section{Results and Discussion}

After our discoveries that butoxyphenyl-NDI crystals ${ }^{12}$ exhibit irreversible thermosalience and reversible thermochromism, we expanded our search to other NDI derivatives based on alkyl chain length. ${ }^{32}$ We hypothesize that longer chains and smaller ring overlaps might flatten the potential energy curve, thus facilitating the transition and improving the reversibility. Accordingly, we observe that this NDI family exhibits thermosalience and/or thermochromism across its many derivatives. Thermosalience is a special case of thermoelastic behavior where the release of accrued elastic stress causes dramatic and often irreversible changes in crystal dimensions. ${ }^{34}$ Upon reaching ten carbons (Decoxyphenyl-NDI; or DNDI for short), we find the crystals are no longer thermosalient, yet they remain thermoelastic, with exceptional rates of thermal cycling and measurable expansion force. What's more, the transition temperature is around $45{ }^{\circ} \mathrm{C}$, a 
temperature range better-suited for near everyday temperature applications than other reversibly thermosalient crystals, which actuate at $>80{ }^{\circ} \mathrm{C}^{35-36}$ or lower than $0{ }^{\circ} \mathrm{C} .{ }^{37}$

Table 1: Crystallographic parameters at $298 \mathrm{~K}$ and $338 \mathrm{~K}$.

\begin{tabular}{|c|c|c|}
\hline Parameter & $298 \mathrm{~K}$ & $338 \mathrm{~K}^{\mathrm{a}}$ \\
\hline Crystal System & \multicolumn{2}{|c|}{ Triclinic } \\
\hline Space Group & \multicolumn{2}{|c|}{$\mathrm{P} \overline{1}$} \\
\hline Formula & \multicolumn{2}{|c|}{$\mathrm{C}_{46} \mathrm{H}_{54} \mathrm{~N}_{2} \mathrm{O}_{6}$} \\
\hline & \multicolumn{2}{|l|}{ Unit cell } \\
\hline $\mathrm{a}(\AA)=$ & $5.232(4)$ & 4.6739 (19) \\
\hline$b(\AA)=$ & $7.643(5)$ & $7.602(2)$ \\
\hline$c(\AA)=$ & 25.120 (18) & 29.218 (10) \\
\hline$\alpha\left(^{\circ}\right)=$ & 86.887 (19) & 98.780 (15) \\
\hline$\beta\left(^{\circ}\right)=$ & 87.346 (19) & $88.17(2)$ \\
\hline$Y\left({ }^{\circ}\right)=$ & $80.28(2)$ & $92.12(6)$ \\
\hline Volume $\left(\AA^{3}\right)$ & 987.8 (12) & $1024.9(6) \AA^{3}$ \\
\hline Crystal Color & \multicolumn{2}{|c|}{ Yellow } \\
\hline Z & 1 & 1 \\
\hline
\end{tabular}

aTo aid in direct comparison between these two polymorphs the unit cell parameters of the $338 \mathrm{~K}$ data have be converted by applying a $3 \times 3$ transformation. See Table S 1 for full details

DNDI was synthesized based on literature protocols, ${ }^{12,32}$ substituting longer alkyl chains, and crystallized from the slow evaporation of chloroform. A detailed synthesis is also available in the SI. These crystals were examined with single-crystal X-ray diffraction at temperatures above and below the transition point, and the single-crystal structure was solved across the operating temperature range. The crystallographic parameters are summarized in Table 1. Structural analysis indicates that the molecular arrangement within the lattice positions align the NDI cores parallel to each other with the alkyl chains fully extended away, forming layers of ribbon-like planes (Figure 1b-1d). The centroid-to-centroid distances-defined by the 16 atoms of the rylene center-between the NDI cores shorten considerably with heating despite little change in the interplanar distances. This is because the NDI centers slide closer together when the crystal's large axis of thermal contraction is arranged horizontally (Figure 1c). This is enabled by a change in the aromatic ring overlap, which causes a contraction of the linkers as temperature decreases. Upon heating past the transition point of $45^{\circ} \mathrm{C}$, the crystal shrinks to $\sim 90 \%$ of its cold length in the long direction (Figure 1e). Put differently, the DNDI crystals were observed to have sudden negative thermal expansion at $45^{\circ} \mathrm{C}$. The width increases slightly, and there is no apparent change in thickness. The hot and cold lengths remain relatively stable (Figure 1f) following multiple cycles without loss in compressibility (Movie S1). Single crystal analysis shows that the cool and warm structures are two different crystal habits, both of which are triclinic, and differ only modestly in unit cell lengths and angles. This small change in packing may explain why the crystals are able to cycle without substantial degradation. 


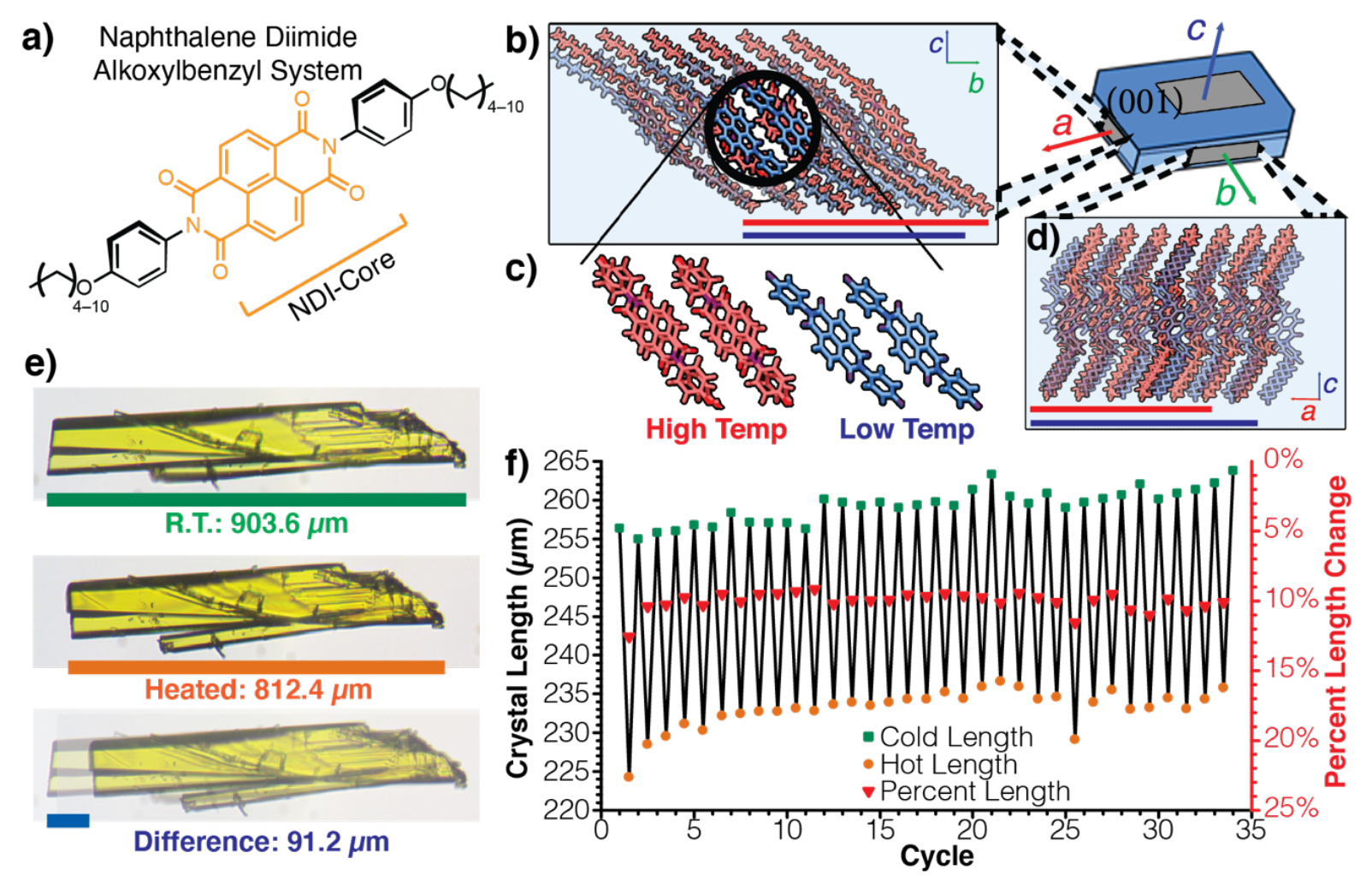

Figure 1. a) The structural formula of the naphtalene diimide (NDI) systems, which all show thermoelastic behavior. When the alkyl chain is $4-9$ carbons long, the crystals are thermosalient. This work shows that when the alkyl chain is 10 carbons long (DNDI), the fluctuation becomes reversible and thermomorphic. Overlap of both crystal phases at high (red) and low (blue) temperatures b) down the crystallographic aaxis, where the expansion along the $b$-axis and $c$-axis from packing is illustrated. c) A Zoom-in into the ring overlaps down the a-axis shows the NDI core and phenoxyl ring overlap becomes slip stacked at high temp and d) down the crystallographic $b$-axis, where the expansion at low temperature is obviated. e) Optical micrographs of a DNDI crystal at room temperature (top), heated (middle) and an overlay illustrating the length difference (bottom). f) Graph showing crystal length changes of a single crystal over 34 thermal cycles from cold (green squares) to hot (orange circles) and back with percent length change (red triangles).

We conducted differential scanning calorimetry on bulk DNDI crystals cycling the temperature from $20{ }^{\circ} \mathrm{C}$ to $60{ }^{\circ} \mathrm{C}$ and back for 200 cycles (Figure 2a). The exothermic and endothermic peaks show no shift in temperature, width, or height over the cycles, indicating that the bulk molecular rearrangement is not attenuated. The recovered crystals were apparently thinner, having slightly delaminated in the DSC though the recovered crystals continued to actuate at the same temperatures with the same $10 \%$ decrease in length. One such recovered crystal is shown in the insert of Figure 2a.

DSC analysis coupled with computational efforts at the Density Functional Theory (DFT) level of theory were employed to give some insight into the driving forces behind the crystal transformation. The endothermic contraction process, which is at its most endothermic at $46.3{ }^{\circ} \mathrm{C}$, occurs with a $\Delta \mathrm{G}$ of $-6.2 \times 10^{3} \mathrm{~J} / \mathrm{mol}$, a $\Delta \mathrm{H}-7.7 \times 10^{3} \mathrm{~J}$, and an entropic penalty of $\Delta \mathrm{S}=-35$ $\mathrm{J} / \mathrm{mol} \bullet \mathrm{C}$. Conversely, the "expansion" process is $\Delta \mathrm{G}$ of $+6.0 \times 10^{3} \mathrm{~J} / \mathrm{mol}$ at $33.3^{\circ} \mathrm{C}$ - the exothermic peak maximum. The reverse process appears largely entropy driven, with a $\Delta \mathrm{S}=59 \mathrm{~J} / \mathrm{mol} \bullet \mathrm{C}$ and a $\Delta \mathrm{H} 8.0 \times 10^{3} \mathrm{~J} / \mathrm{mol}$. The slight discrepancy in free energy between these transitions may arise from the thermal delamination of the crystals. 

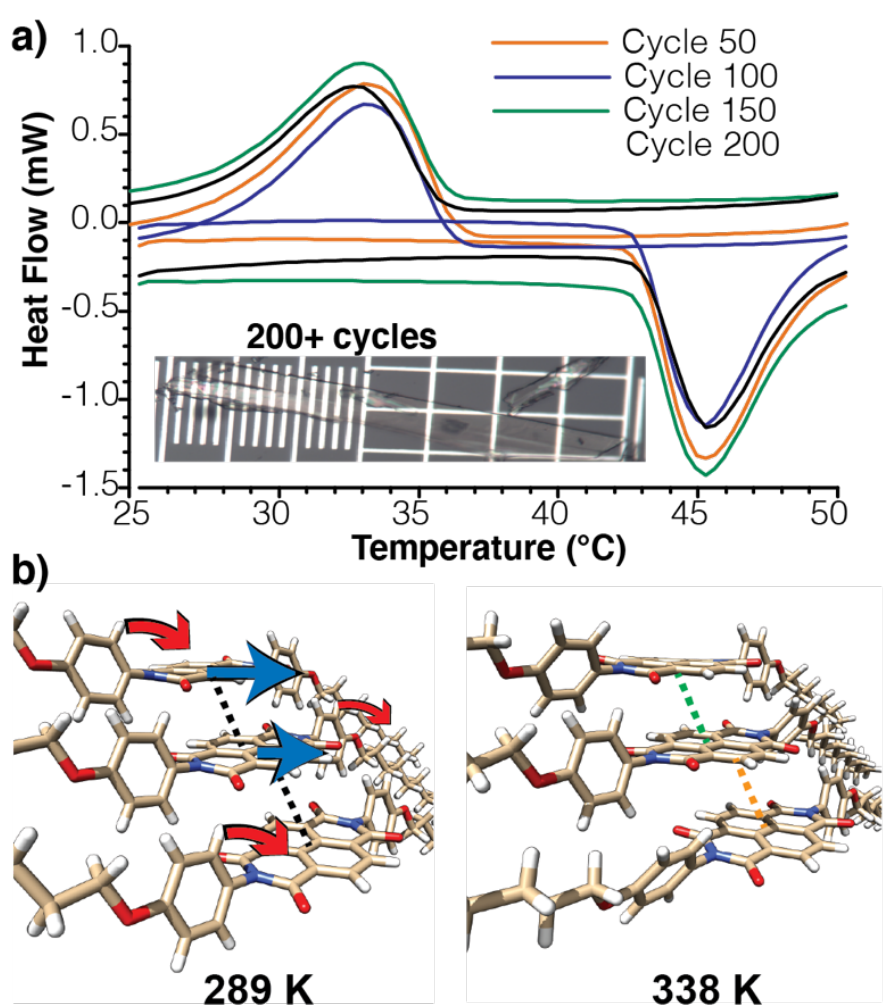

$289 \mathrm{~K}$

$338 \mathrm{~K}$
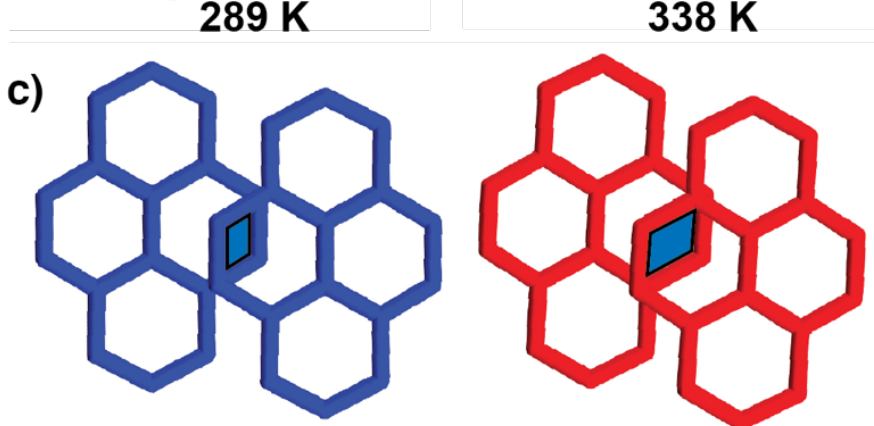

\begin{tabular}{ccccc} 
Temp $(\mathrm{K})$ & $\begin{array}{c}\text { Interplanar } \\
\text { Distance }(\AA)\end{array}$ & $\begin{array}{c}\text { Centroid } \\
\text { Distance }(\AA)\end{array}$ & $\begin{array}{c}\text { Overlap } \\
(\%)\end{array}$ & $\begin{array}{c}\text { Dihedral } \\
\text { Angle }\left(^{\circ}\right)\end{array}$ \\
\hline 298 & 3.316 & 5.232 & $\sim 4$ & 72.29 \\
338 & 3.309 & 4.778 & & 63.34 \\
& & 4.692 & $\sim 12$ & 75.54
\end{tabular}

Figure 2. a) DSC traces of DNDI are shown for $50,100,150$, and 200 cycles. The inset shows a single DNDI crystal from the DSC experiment, which remains $\sim 800 \mu \mathrm{m}$ long, after 207 cycles. Major divisions of the scale are $100 \mu \mathrm{m}$. b) The dynamic motions that DNDIs undergo from the low temperature phase to the high temperature phase involve a rotation of one of the two aromatic rings on each NDI (red arrow) to allow the NDI cores to move over each other (blue arrow). c) While the rotation of the aromatic ring is energetically uphill, the greater pi-overlap in the high temp structure is a lower energy structure at these temperatures with the distances and angles tabulated and color coded from the illustration in panel b.

The DFT calculations were performed considering the periodic crystal structure and molecular model systems (Figure S1, S2). ${ }^{38-39}$ The crystal structure of the DNDI crystal (Figure S1a, S2a), and the molecular clusters models have been used to estimate the different local and non-local effects. More specifically, we studied different molecular models surrounded by 1-3 layers to study the neighboring effect (Figure S1b-e, S2b-e). Thermochemistry calculations were 
performed at $298 \mathrm{~K}$ and $338 \mathrm{~K}$ in all these crystal structures and molecular clusters. Our calculations reveal that $\Delta \mathrm{H}^{\text {theory }}=7.2-8.7 \times 10^{3} \mathrm{~J} / \mathrm{mol}$ when DNDI is surrounded by mono-, bi- and tri-layer molecules. This is excellent in agreement with the experimental value $\left(\Delta \mathrm{H}^{\exp }=7.7-8.0 \times 10^{3}\right.$ $\mathrm{J} / \mathrm{mol})$. The entropy changes from our theoretical calculations is $\Delta \mathrm{S}^{\text {theory }}=53.0-54.3 \mathrm{~J} / \mathrm{mol} \cdot \mathrm{C}$ for the same molecular models, which is in agreement with the experimental values $\left(\Delta S^{\exp }=35-59\right.$ $\mathrm{J} / \mathrm{mol} \cdot \mathrm{C}$ ). In order to understand the barriers to switch from one form to the other, we hypothesize that the driving force for the phase transition is a lower energy co-facial overlap of the NDI cores. This better overlap is inhibited at low temperature by the steric effects of the bulky aromatic rings. This assessment would be consistent with literature that has shown rotational dynamics in the solid state are important factors in a number of thermally responsive systems systems. ${ }^{40}$ Only when there is enough thermal energy for these rings to rotate out of the way, can the NDI cores move closer to each other. Only one ring rotates significantly on each DNDI molecule - the other ring rotates by only a few degrees, which we assumed to have a negligible effect on the energetics. To test this hypothesis, a scan was performed on the rotation of the phenoxy ring per DNDI structure (Figure S3) within repeating stacks modeled from the solid-state data. The estimated electronic rotational barrier is $\Delta \mathrm{E}^{\dagger}=13.6-14.4 \mathrm{~kJ} \cdot \mathrm{mol}^{-1}$, which is not much higher than the available thermal energy at the phases transition. Thus, we postulate that a possible mechanism driving the phase transition is the greater thermal energy to permit partial rotation of the rings. In other words, a full rotation of the rings is not necessary, but the available thermal energy allows for the rings to be perturbed enough to allow for an energetically more favorable $\pi-\pi$ interaction between the NDI cores, which is observed as a decrease in centroid distances. In summary, the overall global motion and experimental thermodynamic data support that an energetically penalizing rotation of every other aromatic ring must occur to allow the NDI cores to slide over each other as depecited in Figure 2b. This rotational dynamics permits greater stabilization from improved overlap of the NDI $\pi$-systems (Figure 2c). These results are consistent with the fact that our $n$-phenoxyl substituted NDI systems containing four, seven, eight, and nine carbons likewise undergo thermosalience. Indeed, more than 820 solid state structures of functionalized NDI cores have been uploaded to the Cambridge Structural Database and many experimentally tested as organic semiconductor systems, yet only the derivatives featuring an $n$-phenoxyl substituent have demonstrated thermoelastic behavior. Therefore, the importance of the aromatic ring and its rotational dynamics cannot be understated. ${ }^{40}$

The single-crystal-to-single-crystal transition occurs with a slight change in unit cell parameters, which contributes to a difference in cellular birefringence. We were able to use this change in birefringence to better understand the mechanism of actuation. During a gradual thermal gradient, the transition occurs slowly, nucleating at a certain point along the crystal long axis while all of the molecules in a line rearrange. The "wavefront" (Figure 3, red diagonal lines) then propagates lengthwise as a solid line in either direction down the crystal (Figure 3, yellow arrows). At crystal imperfections, cracks, or dislocations, the wave front can stall, and the stress lines become apparent. The thermal gradient takes a bit longer to affect the other side of the obstacle, during which the wavefront above or below the disturbance may continue propagating past, causing the crystal to split disjointly until the wave fronts catch up and the sections merge again (Movie S2). The most common method of crystal splitting in this manner is along the plane parallel to the large [001] face, causing delamination of the parallel sheets. Repeated thermal cycles tend to cleave or shear the crystal into thinner crystals that continue to actuate. Intriguingly, the phase transition can occur at any point in the crystal and occasionally at multiple locations. 


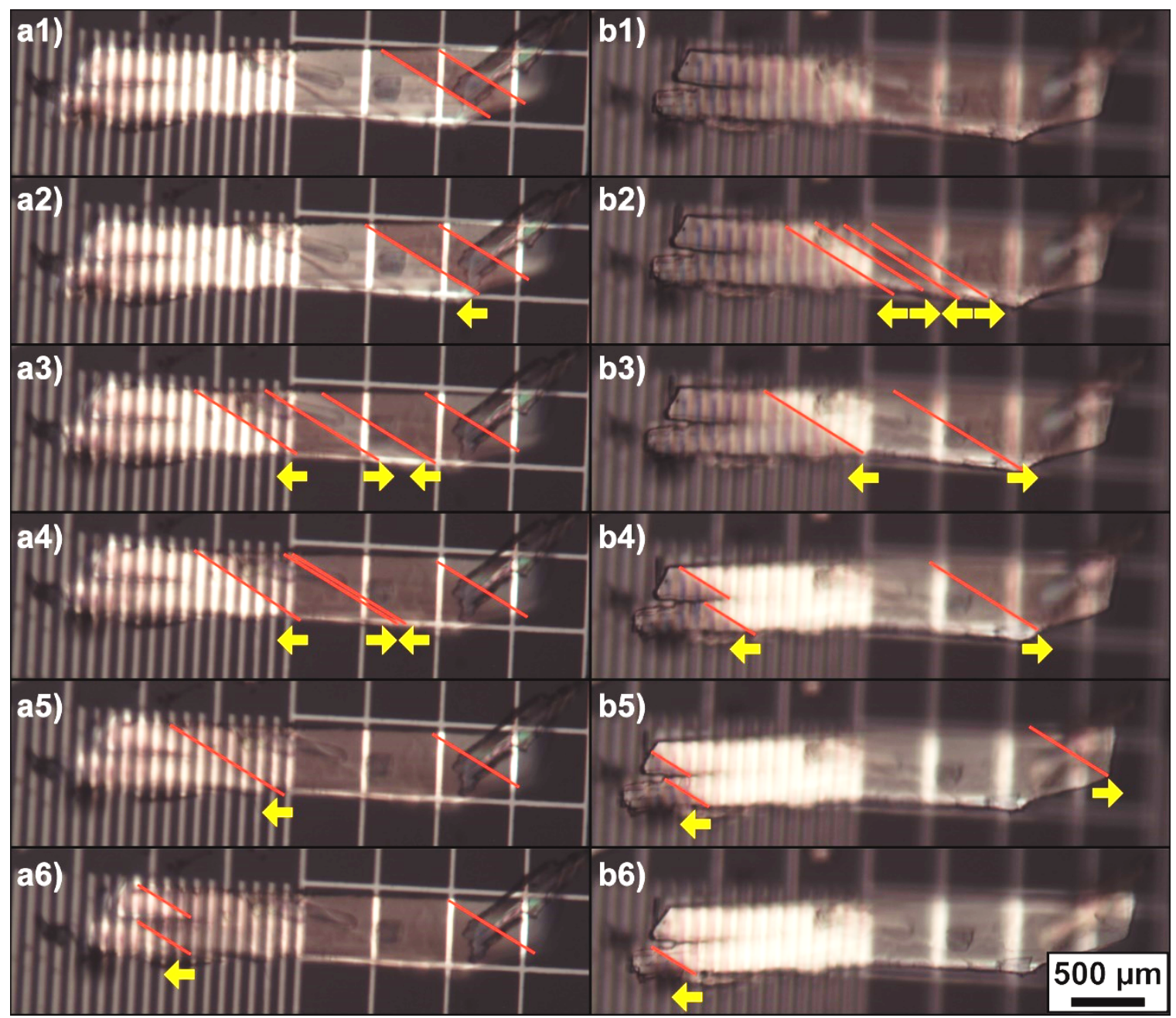

Figure 3. Still frames of DNDI crystal between crossed polarizers set at the extinction position after 200 thermal cycles subjected to a slow thermal gradient. Propagation (yellow arrows) of the molecular rearrangement wavefronts (red lines) through the long axis of the crystal upon a1-6) heating and b1-6) cooling past the respective thermal transition points.

To take advantage of this thermoelasticity, we fabricated a thermoelectric actuator by coating the crystal in a conductive silver layer ${ }^{29}$ for use in an electronic circuit. DNDI exhibits no conductivity in its natural state, so a conductive coating is required to allow current to pass through. Selected large DNDI crystals were metallized via the Tollens Silver Mirror reaction and examined by scanning electron microscopy (SEM) (Figure 4a). A thin uniform coating of silver nanoparticles was found on the crystal surface and the crystals were visually grey and shiny instead of their usual yellow. Attempts to close a circuit with these crystals failed because the silver coating was not thick enough, and the silver mirror reaction was redone on the same crystals. As the silver coating grew thicker, the silver particles merged into a solid sheet. Curiously, once the 
electrothermal motion began the wetted crystal could actuate (Movie S3). However, these crystals successfully closed a circuit and were deemed conductive enough to proceed.

The conductive crystals were connected in an electronic circuit (Figure $4 \mathbf{b}$ ) to bridge a gap of a width such that it is smaller than a cold crystal but larger than a hot one. Initial attempts using copper electrodes resulted in intermittent conduction at best, as there was not very good contact between the crystal and the copper electrodes. Filter paper soaked in saturated sodium chloride as a liquid electrolyte solution was found to work well. Before coating with silver, the DNDI crystals show no electrical conductivity whatsoever, so any electrical measurements are entirely based on the nature of the coating. Upon passing a current through the coated crystal, the silver coating visibly turned black moving from the negative to the positive end of the crystal (Movie S4). Once the current provided enough heat to the circuit path-either the silver coating or the liquid electrolytes - the crystal contracted. Indeed, the crystal, when placed in just the right location, a pulsatile expansion and contraction is observed — several times a second - as the crystal cooled and heated (Movie S5 at approximate the 12 second mark).
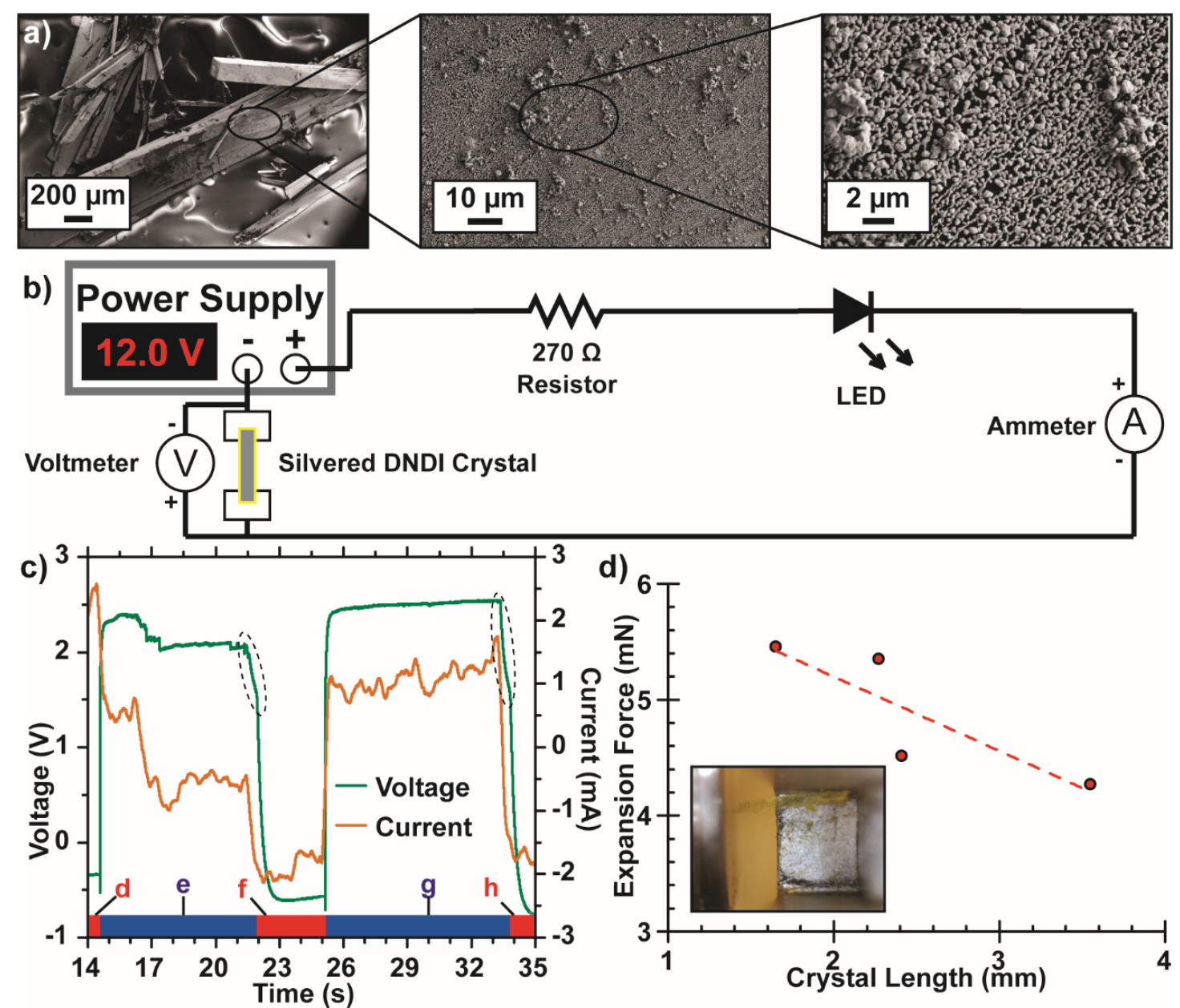

Figure 4. a) Scanning Electron Micrographs of silvered DNDI crystals at progressively higher magnifications. At higher magnification, left-to-right, individual silver nanoparticles are visible. b) Circuit schematic for electrical actuation. c) Oscilloscope traces of the measured voltage and current through the circuit over time. At the bottom, the red sections represent the time the crystal was expanded, and the blue sections represent the time the crystal was contracted. The dotted black ellipses on the voltage trace indicate the capacitor discharge in the power supply upon turning off the voltage. d) Force-length 
comparison of 4 DNDI crystals. The inset shows a crystal (yellow) resting on the aluminum heatsink with the backstop on the right and the sensor on the left.

On applying or removing the voltage, the crystal rapidly responded by contracting or expanding, respectively (Supplemental Movies). If the crystal is placed properly, the heating and cooling induces a pulsive effect as the crystal heats, shrinks, and shorts the circuit. The crystal then cools, expands, and closes the circuit. Analysis of the motion shows that we were able to obtain expansion-re-expansion rates between $1-2 \mathrm{~Hz}$, which is exceptionally rapid for organic crystals. On turning off the power supply, the voltage drops slowly at first, then rapidly to the baseline, indicated by the dotted ellipses in Figure 4c. This is because the capacitors in the power supply are discharging briefly after turning the voltage off.

Re-expansion force measurements were obtained using a piezoelectric sensor placed against a heated crystal and measuring the voltage produced by the piezoelectric material in response to the crystal pushing into it (Figure 4d). This voltage can be converted to force once the physical parameters of the sensor are determined. The crystals selected were difficult to align and register any force, but the force output was plotted against the crystal length for four crystals in a range of $\sim 1.5$ to $\sim 3.5 \mathrm{~mm}$. The forces registered in a range of $\sim 4$ to $\sim 5.5 \mathrm{mN}$ show a loose inverse proportion with length. However, the crystals tend to break apart under resistance to expansion. This suggests that the internal forces holding the [001] planes together are weaker than the outward expansion force, as the energy required to push outwards instead shears the crystal along its plane.

\section{Conclusion}

In summary, we have shown that DNDI can be used as a thermally activated actuator for use as an electrical switch or indicator, reusable over many cycles in ambient and near physiologically important temperatures. These crystals retain their actuation capability even after breaking into smaller parts and show promising potential for use embedded in an elastic matrix that can align and magnify the expansive capabilities of DNDI for use as a thermal-actuated sensor, muscle, extracting energy in the diurnal cycle of desert climates, or in thermomechanical automatic devices. These crystals have potential to impact the fields of electronics and micromechanics in different directions.

\section{Experimental}

\section{Synthesis of DNDI}

DNDI was synthesized based on literature protocols, ${ }^{32,41}$ purified by silica column chromatography in isocratic chloroform and crystallized by evaporation from the fraction tubes.

\section{Metallization of DNDI}

Sufficiently large DNDI crystal candidates were selected and added to a scintillation vial with 5 $\mathrm{mL}$ silver nitrate, $200 \mathrm{mg}$ glucose, and 5 drops ammonium hydroxide, and gently heated while swirling to around $50^{\circ} \mathrm{C}$ until the silver mirror began to form. The vial was then left on a rotisserie at room temperature for 2 days and then vacuum filtered while rinsing with ultrapure water and drying.

\section{Circuit Design}

A circuit was set up and soldered to a breadboard consisting of a terminal for power supply, a 270 $\Omega$ resistor, a red LED, terminals for current measurement, electrodes for the crystal switch 
contacts, and a terminal for return power supply. The crystal switch contacts consisted of filter paper strips moistened by saturated sodium chloride solution and mounted above acrylic blocks with an air gap for the crystal to bridge without electrolytes causing a short circuit. The LED indicated a closed circuit, the resistor protected the LED from overcurrent, and the current was monitored as the power supply voltage was increased until the crystal actuated. The metallized crystal actuation was monitored with a USB microscope.

\section{Force Measurements}

Prior to coating with silver, crystals were placed between the fins of an aluminum heatsink with a machined steel rod as an adjustable backstop on one end and a Honeywell FSG005WNPB force sensor on the other, and a thermocouple glued to the outside of the fins. The system was heated by direct contact with a soldering iron tip at $800{ }^{\circ} \mathrm{C}$ until the crystal contracted. The force sensor and backstop were adjusted such that the crystal was butted up against both and would press against the sensor upon re-expansion. The system was then cooled by direct contact with an ice cube on the underside of the heatsink until the crystal expanded into the force sensor. The voltage change was recorded and converted to force according to the sensor properties listed in the manufacturer's datasheet.

\section{Computational Details:}

Due to the extended nature of the bulk DNDI crystal structure, the periodic crystal structures (Figure S1a, S2b) and four cluster modeling systems with 1-4 layers were used (Figure S1b-d, S2b-d). The equilibrium structures, geometries, calculations of energetics and rotational barrier of the model systems of the DNDI crystal were obtained with the hybrid unrestricted dispersioncorrected density functional theory (DFT) method noted by UB3LYP-D3. The correlationconsistent polarization double $\zeta$-quality basis sets was used for all atoms. ${ }^{38-39}$ The vibrational frequencies at the optimized geometries were analyzed to confirm ground states. The thermochemical calculations were performed at $298 \mathrm{~K}$ and $338 \mathrm{~K}$ corresponding to each crystal structure configuration. The rigid scan was performed by considering the same models (Figure S3). The threshold used for evaluating the convergence of the energy, forces, and electron density was $10^{-7}$ a.u. for each parameter. All calculations were performed with the Gaussian09 quantum chemistry code. ${ }^{42}$ The details of the computations can be found in the Supporting Information.

\section{Associated Content}

Supplementary Information containing experimental and computational details and supplemental movies S1-S4 are available online at [future website]

Crystal structure data that support the findings of this study have been deposited in the CCDC with the accession codes CCDC 1992519-1992523. These data can be obtained free of charge via www.ccdc.cam.ac.uk/data_request/cif, or by emailing data_request@eccdc.cam.ac.uk, or by contacting The Cambridge Crystallographic Data Centre, 12 Union Road, Cambridge CB2 1EZ, UK; fax: +44 1223336033 . 


\section{Acknowledgements}

J.J.G. would like to acknowledge National Science Foundation [CAREER DMR-1654405 and DMR-2003534], the Welch Foundation [AT-1989-20190330], and the ACS Petroleum Research Fund [57627-DNI10] S. P. acknowledges the support from the SERB-DST, Government of India for providing the Ramanujan Faculty Fellowship under the Grant No. SB/S2/RJN-067/2017 and Early Career Research Award (ECRA) under the Grant No. ECR/2018/000255.

ORCID: Dr Srimanta Pakhira 0000-0002-2488-300X.

Dr. Jose L. Mendoza-Cortes: 0000-0001-5184-1406.

\section{Author Information}

The authors declare no competing interests. ${ }^{\dagger}$ These authors contributed equally to this work.

Correspondence and requests for materials should be addressed to gassensmith@utdallas.edu or jmendoza@msu.edu

\section{References}

1. Otero, T. F.; Sansieña, J. M., Soft and Wet Conducting Polymers for Artificial Muscles. Adv Mater 1998, 10 (6), 491-494.

2. Okuzaki, H.; Osada, Y., Electro-driven chemomechanical polymer gel as an intelligent soft material. J Biomater Sci Polym Ed 1994, 5 (5), 485-496.

3. Seki, T.; Sakurada, K.; Muromoto, M.; Ito, H., Photoinduced single-crystal-to-singlecrystal phase transition and photosalient effect of a gold(i) isocyanide complex with shortening of intermolecular aurophilic bonds. Chem Sci 2015, 6 (2), 1491-1497.

4. Peterson, G. I.; Dobrynin, A. V.; Becker, M. L., Biodegradable Shape Memory Polymers in Medicine. Adv Healthc Mater 2017, 6 (21), 1700694.

5. Ge, F.; Zhao, Y., A new function for thermal phase transition-based polymer actuators: autonomous motion on a surface of constant temperature. Chem Sci 2017, 8 (9), 6307-6312.

6. Zhao, Q.; Zou, W.; Luo, Y.; Xie, T., Shape memory polymer network with thermally distinct elasticity and plasticity. Science Advances 2016, 2 (1), e1501297.

7. Wang, J.; Zhao, Q.; Cui, H.; Wang, Y.; Chen, H.; Du, X., Tunable shape memory polymer mold for multiple microarray replications. J Mat Chem A 2018, 6 (48), 24748-24755.

8. Zhao, Q.; Wang, Y.; Cui, H.; Du, X., Bio-inspired sensing and actuating materials. J Mater Chem C 2019, 7 (22), 6493-6511.

9. Omoto, K.; Nakae, T.; Nishio, M.; Yamanoi, Y.; Kasai, H.; Nishibori, E.; Mashimo, T.; Seki, T.; Ito, H.; Nakamura, K.; Kobayashi, N.; Nakayama, N.; Goto, H.; Nishihara, H., Thermosalience in Macrocycle-Based Soft Crystals via Anisotropic Deformation of Disilanyl Architecture. J Am Chem Soc 2020, 142 (29), 12651-12657.

10. Ding, X.; Unruh, D. K.; Groeneman, R. H.; Hutchins, K. M., Controlling thermal expansion within mixed cocrystals by tuning molecular motion capability. Chem Sci 2020, 11 (29), 77017707. 
11. Panda, M. K.; Runčevski, T.; Chandra Sahoo, S.; Belik, A. A.; Nath, N. K.; Dinnebier, R. E.; Naumov, P., Colossal positive and negative thermal expansion and thermosalient effect in a pentamorphic organometallic martensite. Nat Commun 2014, 5, 4811.

12. Dharmarwardana, M.; Welch, R. P.; Kwon, S.; Nguyen, V. K.; McCandless, G. T.; Omary, M. A.; Gassensmith, J. J., Thermo-mechanically responsive crystalline organic cantilever. Chem Commun 2017, 53 (71), 9890-9893.

13. Panda, M. K.; Centore, R.; Causà, M.; Tuzi, A.; Borbone, F.; Naumov, P., Strong and Anomalous Thermal Expansion Precedes the Thermosalient Effect in Dynamic Molecular Crystals. Scientific Reports 2016, 6, 29610.

14. Chung, H.; Chen, S.; Sengar, N.; Davies, D. W.; Garbay, G.; Geerts, Y. H.; Clancy, P.; Diao, Y., Single Atom Substitution Alters the Polymorphic Transition Mechanism in Organic Electronic Crystals. Chem Mater 2019, 31 (21), 9115-9126.

15. Pathigoolla, A.; Sureshan, K. M., Synthesis of triazole-linked homonucleoside polymers through topochemical azide-alkyne cycloaddition. Angew Chem Int Ed Engl 2014, 53 (36), 95225 .

16. Lauher, J. W.; Fowler, F. W.; Goroff, N. S., Single-crystal-to-single-crystal topochemical polymerizations by design. Acc Chem Res 2008, 41 (9), 1215-29.

17. Pathigoolla, A.; Gonnade, R. G.; Sureshan, K. M., Topochemical click reaction: spontaneous self-stitching of a monosaccharide to linear oligomers through lattice-controlled azide-alkyne cycloaddition. Angew Chem Int Ed Engl 2012, 51 (18), 4362-6.

18. Barbour, L. J., Single Crystal to Single Crystal Transformations. Aust J Chem 2006, 59 (9), 595.

19. Naumov, P.; Chizhik, S.; Panda, M. K.; Nath, N. K.; Boldyreva, E., Mechanically Responsive Molecular Crystals. Chem Rev 2015, 115 (22), 12440-90.

20. Burgert, I.; Fratzl, P., Actuation systems in plants as prototypes for bioinspired devices. Philos Trans A Math Phys Eng Sci 2009, 367 (1893), 1541-57.

21. Balzani, V.; Credi, A.; Raymo, F. M.; Stoddart, J. F., Artificial Molecular Machines. Angew Chem 2000, 39 (19), 3348-3391.

22. Kobatake, S.; Takami, S.; Muto, H.; Ishikawa, T.; Irie, M., Rapid and reversible shape changes of molecular crystals on photoirradiation. Nature 2007, 446 (7137), 778-81.

23. Morimoto, M.; Irie, M., A diarylethene cocrystal that converts light into mechanical work. J Am Chem Soc 2010, 132 (40), 14172-8.

24. Medishetty, R.; Sahoo, S. C.; Mulijanto, C. E.; Naumov, P.; Vittal, J. J., Photosalient Behavior of Photoreactive Crystals. Chem Mater 2015, 27 (5), 1821-1829.

25. Liu, G.; Liu, J.; Ye, X.; Nie, L.; Gu, P.; Tao, X.; Zhang, Q., Self-Healing Behavior in a Thermo-Mechanically Responsive Cocrystal during a Reversible Phase Transition. Angew Chem Int Ed Engl 2017, 56 (1), 198-202.

26. Lusi, M.; Bernstein, J., On the propulsion mechanism of "jumping" crystals. Chem Commun (Camb) 2013, 49 (81), 9293-5.

27. Panda, M. K.; Runcevski, T.; Husain, A.; Dinnebier, R. E.; Naumov, P., Perpetually selfpropelling chiral single crystals. J Am Chem Soc 2015, 137 (5), 1895-902.

28. Nath, N. K.; Panda, M. K.; Sahoo, S. C.; Naumov, P., Thermally induced and photoinduced mechanical effects in molecular single crystals - a revival. CrystEngComm 2014, 16 (10), 1850.

29. Khalil, A.; Ahmed, E.; Naumov, P., Metal-coated thermosalient crystals as electrical fuses. Chem Commun 2017, 53 (60), 8470-8473. 
30. Jin, M.; Yamamoto, S.; Seki, T.; Ito, H.; Garcia-Garibay, M. A., Anisotropic Thermal Expansion as the Source of Macroscopic and Molecular Scale Motion in Phosphorescent Amphidynamic Crystals. Angew Chem Int Ed 2019, 58 (50), 18003-18010.

31. Chung, H.; Dudenko, D.; Zhang, F.; D’Avino, G.; Ruzié, C.; Richard, A.; Schweicher, G.; Cornil, J.; Beljonne, D.; Geerts, Y.; Diao, Y., Rotator side chains trigger cooperative transition for shape and function memory effect in organic semiconductors. Nat Commun 2018, 9 (1), 278.

32. Dharmarwardana, M.; Arimilli, B. S.; Luzuriaga, M. A.; Kwon, S.; Lee, H.; Appuhamillage, G. A.; McCandless, G. T.; Smaldone, R. A.; Gassensmith, J. J., The thermoresponsive behavior in molecular crystals of naphthalene diimides and their 3D printed thermochromic composites. CrystEngComm 2018, 20 (39), 6054-6060.

33. Dewhirst, M. W.; Viglianti, B. L.; Lora-Michiels, M.; Hoopes, P. J.; Hanson, M., THERMAL DOSE REQUIREMENT FOR TISSUE EFFECT: EXPERIMENTAL AND CLINICAL FINDINGS. Proc SPIE Int Soc Opt Eng 2003, 4954, 37.

34. Park, S. K.; Diao, Y., Martensitic transition in molecular crystals for dynamic functional materials. Chem Soc Rev 2020, 49 (22), 8287-8314.

35. Alimi, L. O.; van Heerden, D. P.; Lama, P.; Smith, V. J.; Barbour, L. J., Reversible thermosalience of 4-aminobenzonitrile. Chem Commun 2018, 54 (48), 6208-6211.

36. Lončarić, I.; Popović, J.; Despoja, V.; Burazer, S.; Grgičević, I.; Popović, D.; Skoko, Ž., Reversible Thermosalient Effect of N'-2-Propylidene-4-hydroxybenzohydrazide Accompanied by an Immense Negative Compressibility: Structural and Theoretical Arguments Aiming toward the Elucidation of Jumping Phenomenon. Cryst Growth Des 2017, 17 (8), 4445-4453.

37. Karothu, D. P.; Weston, J.; Desta, I. T.; Naumov, P., Shape-Memory and Self-Healing Effects in Mechanosalient Molecular Crystals. Journal of the American Chemical Society 2016, 138 (40), 13298-13306.

38. Grimme, S.; Antony, J.; Ehrlich, S.; Krieg, H., A consistent and accurate ab initio parametrization of density functional dispersion correction (DFT-D) for the 94 elements H-Pu. $J$ Chem Phys 2010, 132 (15), 154104.

39. Becke, A. D., Density-functional thermochemistry. III. The role of exact exchange. JChem Phys 1993, 98 (7), 5648-5652.

40. Hutchins, K. M.; Groeneman, R. H.; Reinheimer, E. W.; Swenson, D. C.; MacGillivray, L. R., Achieving dynamic behaviour and thermal expansion in the organic solid state via cocrystallization. Chem Sci 2015, 6 (8), 4717-4722.

41. Fomine, S.; Fomina, L.; Arreola, R.; Alonso, J., Bisimide-lactamimide ring contraction in six-membered polyimides. Polymer 1999, 40 (8), 2051-2058.

42. Frisch, M. J.; Trucks, G. W.; Schlegel, H. B.; Scuseria, G. E.; Robb, M. A.; Cheeseman, J. R.; Scalmani, G.; Barone, V.; Petersson, G. A.; Nakatsuji, H.; Li, X.; Caricato, M.; Marenich, A. V.; Bloino, J.; Janesko, B. G.; Gomperts, R.; Mennucci, B.; Hratchian, H. P.; Ortiz, J. V.; Izmaylov, A. F.; Sonnenberg, J. L.; Williams; Ding, F.; Lipparini, F.; Egidi, F.; Goings, J.; Peng, B.; Petrone, A.; Henderson, T.; Ranasinghe, D.; Zakrzewski, V. G.; Gao, J.; Rega, N.; Zheng, G.; Liang, W.; Hada, M.; Ehara, M.; Toyota, K.; Fukuda, R.; Hasegawa, J.; Ishida, M.; Nakajima, T.; Honda, Y.; Kitao, O.; Nakai, H.; Vreven, T.; Throssell, K.; Montgomery Jr., J. A.; Peralta, J. E.; Ogliaro, F.; Bearpark, M. J.; Heyd, J. J.; Brothers, E. N.; Kudin, K. N.; Staroverov, V. N.; Keith, T. A.; Kobayashi, R.; Normand, J.; Raghavachari, K.; Rendell, A. P.; Burant, J. C.; Iyengar, S. S.; Tomasi, J.; Cossi, M.; Millam, J. M.; Klene, M.; Adamo, C.; Cammi, R.; Ochterski, J. W.; Martin, R. L.; Morokuma, K.; Farkas, O.; Foresman, J. B.; Fox, D. J. Gaussian 16 Rev. E.01, Gaussian, Inc.: Wallingford, CT, 2016. 
